\title{
CUARTO SONDEO DE OPINIÓN CIUDADANA DE INSTITUCIONES, AUTORIDA- DES Y ASPECTOS LOCALES EN LA ZONA URBANA DEL CANTÓN AMBATO
}

Fernando Mayorga' Tatiana Vayas ${ }^{2}$ Carolina Freire ${ }^{3}$ Lorena Rivera ${ }^{4}$

Pag 10-15

\section{Resumen}

En este artículo se presentan los resultados del cuarto sondeo - de opinión ciudadana realizado en la zona urbana del cantón Ambato para determinar el nivel de imagen de sus instituciones y la percepción sobre la credibilidad de las autoridades de la provincia de Tungurahua, así como también medir, a través del índice de percepción ciudadana, aspectos locales relacionados con servicios básicos, seguridad vial, calidad del transporte público, seguridad ciudadana e instituciones de educación superior.

\section{Palabras clave:}

Índice, opinión, percepción ciudadana, sondeo.

\begin{abstract}
This research shows the results of the fourth citizen opinion probe that was done in the urban area of Ambato to determine the image level of its institutions and the perception about the credibility of Tungurahua's province authorities, as well as, the information as used to measure local aspects related to basic services, road security, public transportation quality, citizen security and higher education institutions through the perception citizen index.
\end{abstract}

\section{Keywords:}

Index, opinion, citizen perception, probe.

\section{Introducción}

El Observatorio Económico y Social de Tungurahua - OBEST, en su rol de generar indicadores trascendentales de fuente primaria de la provincia que contribuyan como herramienta en la toma de decisiones, realiza sondeos de opinión ciudadana.

Desde diciembre de 2014 el OBEST ha venido aplicando semestralmente el sondeo de opinión ciudadana sobre instituciones, autoridades y aspectos locales en la zona urbana del cantón Ambato, con la finalidad de dar seguimiento continuo a la evolución del índice de percepción sobre autoridades e instituciones.

El sondeo de opinión ciudadana proporciona información útil para que las instituciones y autoridades evaluadas del cantón Ambato, conozcan la percepción que tiene la ciudadanía sobre ellos y los ayuden a tomar decisiones en beneficio de la población. De igual manera, esta información es presentada al público en general para que conozcan aspectos de su ciudad y la percepción que tienen del entorno en el que viven.

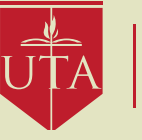

UNIVERSIDAD

TÉCNICA DE AMBATO

\footnotetext{
Ingeniero en Ciencias Económicas y Financieras - Analista en Investigación y Desarrollo del OBEST UTA. fa.mayorga@uta.edu.ec

2Economista - Analista en Investigación y Desarrollo del OBEST-UTA. tI.vayas@uta.edu.ec

${ }^{3}$ Ingeniera Comercial - Analista en Investigación y Desarrollo del OBEST-UTA. Ic.freire@uta.edu.ec ${ }^{4}$ Doctora en Ciencias de la Empresa - Economía y Finanzas - Especialista en Investigación y Desarrollo del OBEST-UTA. pl.rivera@uta.edu.ec
}

Fecha de recepción: 5 Diciembre 2016

Fecha de aceptación: 30 Diciembre 2016

\section{Metodología}

A partir del 26 de octubre del presente año, se realizó el levantamiento de información para la cuarta ronda del sondeo de opinión ciudadana sobre instituciones, autoridades y aspectos locales en la zona urbana del cantón Ambato por parte del equipo técnico del OBEST de la Universidad Técnica de Ambato - UTA, con una duración de 4 días calendario.

La elaboración del sondeo toma tres modelos como base de investigación: Centro de Estudios Sociales y de Opinión Pública de México, denominado "Confianza en las instituciones, México en perspectiva comparada". (Moreno, A., 2010:37). Estudio del Salvador del Instituto Universitario de Opinión Pública - IUDOP, de la Universidad Centroamericana José Simeón Cañas - UCA sobre "La percepción de la seguridad y la confianza en instituciones públicas. Línea de base del plan de acción asocio para el crecimiento". (Aguilar, J., 2013:41-51). Estudio de IBOPE inteligencia sobre el Índice de Confianza Social Chile - 1ra medición 2011.

El universo objeto de estudio corresponde a los 165.185 pobladores que residen en la zona urbana del cantón Ambato (INEC Censo 2010). La muestra calculada es de 630 personas a quienes se aplica la encuesta de acuerdo a la estratificación de 49 zonas urbanas censales determinadas por el INEC (2010), con un coeficiente de confiabilidad del $95 \%$ y un error probable del $4 \%$.

En el análisis se evidencia que el $56 \%$ de los encuestados fueron mujeres y el $44 \%$ hombres. El $42 \%$ de los encuestados tienen estudios de bachillerato, el 33\% posee educación superior, el 22\% educación básica, el 1\% posgrado y el 1\% ningún nivel de instrucción.

En esta cuarta ronda de investigación se mantienen las preguntas de percepción sobre la imagen de las instituciones; credibilidad de autoridades; la calidad del suministro de los servicios; transporte público; uso de fotorradares; actos de-

La base del sondeo es ponderar la percepción, la misma que es un proceso subjetivo, selectivo y temporal, porque inciden intereses y actitudes personales, la experiencia, expectativas y las demandas de tipo temporal situacional o social. (Sánchez, R. 2014:49)

lincuenciales; y, la opinión que tienen los ciudadanos urbanos sobre las universidades.

En esta investigación se eliminaron las preguntas respecto a matriculación vehicular, sector cooperativo y control de velocidad; además se incorporó como institución al Consejo Nacional Electoral - CNE y como servicio al parqueo tarifado para su evaluación.

La variable edad, permitió evidenciar un promedio de 43,5 años para el total de informantes, edad máxima de 73,5 años y mínima de 23,5.

A continuación se presenta el contenido del artículo en cuatro secciones de Ambato y la provincia de Tungurahua: 
Fernando Mayorga,Tatiana Vayas, Carolina Freire, Lorena Rivera/ Cuarto sondeo de opinión ciudadana de instituciones, autoridades y aspectos locales en la zona urbana del cantón Ambato

1. Imagen de las instituciones

2. Credibilidad de las autoridades

3. Aspectos locales

- Calidad de los suministros públicos

- Calidad del servicio de transporte público

- Uso de fotorradares y seguridad vial

- Actos delincuenciales

4. Imagen de las Universidades

\section{Contenido}

\section{Imagen de las Instituciones de Ambato y la provincia de Tungurahua}

Se pregunta a los encuestados de acuerdo a su precepción, cuál es la cualidad que asignan a las instituciones públicas y privadas que operan en Ambato, para lo cual tienen cuatro opciones de respuesta: "muy buena"; "buena"; "regular" y "mala", con respecto a la imagen de doce entidades que se detalla en la Tabla 1.

Tabla 1. Percepción ciudadana sobre imagen institucional en la zona urbana del cantón Ambato (\% encuestados) Octubre 2016

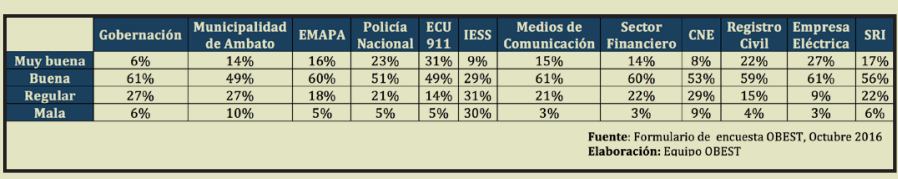

De las doce instituciones evaluadas, la opinión de los ciudadanos ambateños sobre la imagen de las instituciones públicas y privadas se detalla a continuación:

Tabla 2. Instituciones de Ambato con puntuaciones significativas Octubre 2016

\begin{tabular}{|c|c|}
\hline Escala & Puntuaciones significativas \\
\hline Muy buena & ECU 911 \\
\hline Buena & $\begin{array}{c}\text { Gobernación } \\
\text { Medios de comunicación } \\
\text { EEASA }\end{array}$ \\
\hline Regular & IESS \\
\hline Mala & IESS \\
\hline
\end{tabular}

Con el fin de realizar una comparación de los resultados del segundo, tercer y cuarto sondeo de opinión ciudadana, se presenta el análisis que relaciona el índice de percepción ciudadana sobre imagen institucional aplicada en junio 2015, marzo 2016 y octubre 2016 evidenciando los resultados en la figura 1.

Es importante indicar que en este apartado existen diferencias entre la segunda, tercera y cuarta ronda, específicamente en lo que respecta a las instituciones a ser evaluadas y para un mejor análisis, se detalla en la tabla 3.

\section{Tabla 3. Detalle de instituciones evaluadas en la II, III y IV rondas} del sondeo 2015-2016

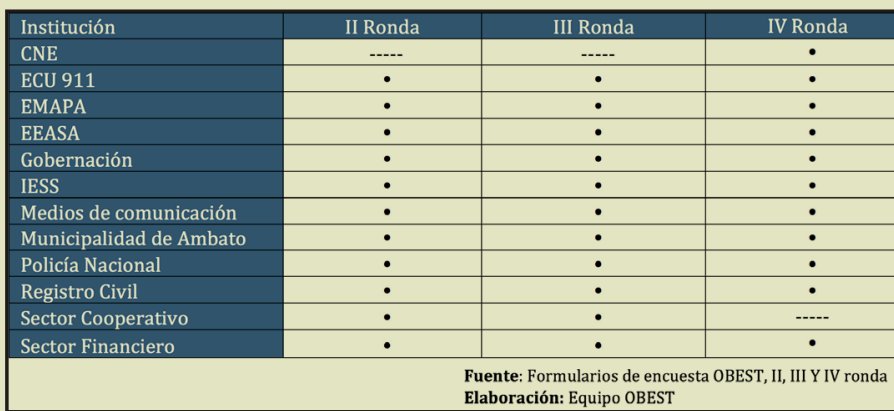

El índice de percepción ciudadana de la imagen institucional se elabora con base a las frecuencias de respuesta descritas en la figura 1, las que se ponderan en una escala de 0 a 100 puntos.

El índice de percepción ciudadana sobre la imagen institucional es la descripción cuantitativa de las cualidades funcionales y atributos psicológicos que el público mentalmente atribuye a la empresa o institución y no es una cualidad inherente a la organización.

(Muñoz, M., 2010: 165)

Cabe recalcar que el índice no mide ni evalúa la gestión y desempeño de estos establecimientos. Con este antecedente se puede presentar el ranking de percepción de imagen.

Figura 1. Índice de Percepción ciudadana sobre imagen institucional en la zona urbana del cantón Ambato (escala: 0 a 100 puntos) II, III y IV ronda del sondeo

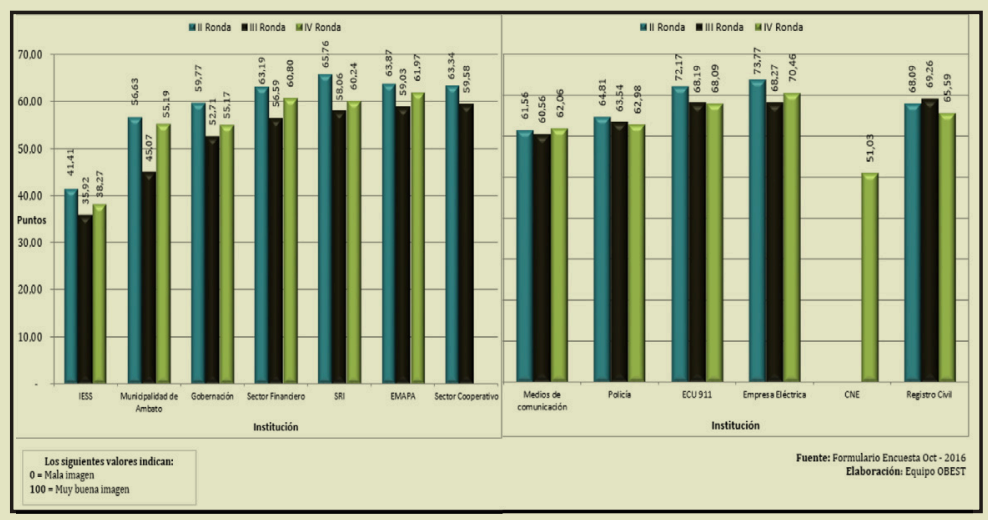

Se expone el análisis de las instituciones en tres dimensiones: 1) mejor puntuada; 2) menor puntuada; y, 3) varianza entre valoraciones obtenidas en la tercera y cuarta ronda.

Primera dimensión: Los datos sitúan a la Empresa Eléctrica (EEASA) con 70,46 puntos, siendo esta la mejor puntuada de las doce instituciones evaluadas, por tanto, se evidencia una percepción positiva de los ambateños a la imagen que proyecta esta entidad en un contexto general.

La comparativa en esta dimensión muestra que en la cuarta ronda, la Empresa Eléctrica lidera el grupo de instituciones evaluadas (70,46 puntos), reemplazando al Registro Civil que en la tercera ronda ocupó el primer puesto (69,26 puntos), tomando en cuenta que los sujetos encuestados son quienes asignan atributos o cualidades a una institución derivadas de su vivencia.

Segunda dimensión: La puntuación más baja tiene el IESS con 38,27 puntos, reflejando la opinión negativa de los sujetos encuestados, respecto a esta institución. 
Manteniendo el mismo esquema de análisis que la dimensión anterior, respecto a la comparativa entre la tercera y cuarta ronda, el IESS se mantiene en el último puesto en el ranking de percepción.

Tercera dimensión: Los datos de la cuarta ronda, muestran que ocho de las doce instituciones evaluadas registran una tendencia creciente con respecto a los resultados de la tercera ronda.

La Municipalidad de Ambato es la institución que refleja un mayor crecimiento (22,47\%), es decir, los ciudadanos ambateños opinan positivamente de este organismo.

Por lo contrario, al analizar las puntuaciones decrecientes de la imagen de las instituciones entre la tercera y cuarta ronda, la variación negativa con mayor peso, es para el Registro Civil $(-5,30 \%)$, datos que la desplazaron del primer al tercer puesto en el ranking de percepción.

\section{Credibilidad de las autoridades de Ambato y la provincia de Tungurahua}

Para efectos de medir la percepción de los sujetos encuestados sobre la credibilidad de las autoridades de Ambato y la provincia de Tungurahua se formuló una interrogante con alternativas de selección de "mucha", "algo", "poco" y "nada". En la cual el Prefecto de la provincia de Tungurahua obtuvo la mayoría de respuestas en el nivel de "mucha credibilidad" entre las cinco autoridades consideradas en este sondeo.

Tabla 4. Percepción ciudadana sobre credibilidad de autoridades locales en la zona urbana del cantón Ambato (\% encuestados) Octubre 2016

\begin{tabular}{|l|c|c|c|c|c|}
\hline & Alcalde & Prefecto & Gobernadora & $\begin{array}{c}\text { Concejales de } \\
\text { Tungurahua }\end{array}$ & $\begin{array}{c}\text { Asambleistas } \\
\text { de Tungurahua }\end{array}$ \\
\hline Mucha credibilidad & $10 \%$ & $26 \%$ & $12 \%$ & $5 \%$ & $3 \%$ \\
\hline Algo de credibilidad & $33 \%$ & $33 \%$ & $33 \%$ & $26 \%$ & $24 \%$ \\
\hline Poca credibilidad & $33 \%$ & $29 \%$ & $36 \%$ & $44 \%$ & $37 \%$ \\
\hline Nada de credibilidad & $23 \%$ & $12 \%$ & $19 \%$ & $25 \%$ & $36 \%$ \\
\hline \multicolumn{7}{|c|}{} & $\begin{array}{c}\text { Fuente: Formulario de encuesta OBEST, Octubre 2016 } \\
\text { Elaboración: Eauivo OBEST }\end{array}$ \\
\hline
\end{tabular}

La comparación del índice de credibilidad de autoridades locales en la segunda, tercera y cuarta rondas del sondeo (junio 2015, marzo 2016 y octubre 2016).

Los datos de la segunda y tercera ronda presentan un escenario a la baja para todas las dignidades, donde el orden del ranking del tercer sondeo, de primero a quinto lugar, es: Prefecto de la provincia de Tungurahua, Gobernadora, Concejales del cantón Ambato, Asambleístas de Tungurahua; y, finalmente Alcalde de Ambato.

En la cuarta ronda, la tendencia es creciente para todas las autoridades y la tabla de posicionamiento se modifica, teniendo como resultado el siguiente orden, de primero a quinto lugar: Prefecto de la provincia de Tungurahua, El índice de percepción ciudadana sobre la credibilidad de autoridades, indica que una persona es creíble cuando posee en sí atributos que le hacen acreedora de la confianza de las personas, a la aprobación de su ejemplo, discurso y representatividad en su cargo, (Burgoa, L., 1995: 49) mas no evalúa a la persona ni su desempeño en sus funciones laborales.

Gobernadora, Alcalde de Ambato, Concejales; y, finalmente Asambleístas de Tungurahua.

Respecto a la variación creciente de la credibilidad a las autoridades que manifiestan los ciudadanos ambateños, se hace una com- paración al índice calculado entre los datos de la tercera y cuarta ronda.

En primer lugar se ubica el Prefecto de la provincia de Tungurahua con un índice de 56,99 puntos, registra un incremento de 6,42 puntos; seguido de la Gobernadora con 45,11 puntos (sube 2,34 puntos); en tercer lugar se encuentra el Alcalde de Ambato con 43,05 puntos, su crecimiento es de 16,12 puntos; la cuarta posición tienen los Concejales del cantón Ambato con 36,14 puntos, esto es 5,87 puntos más que en el tercer sondeo; y, finalmente están los Asambleístas de Tungurahua con 31,15 puntos con un aumento de 1,77 puntos.

Figura 2. Índice de Percepción ciudadana sobre credibilidad de autoridades locales en la zona urbana del cantón Ambato (escala: 0 a 100 puntos) II, III y IV ronda del sondeo

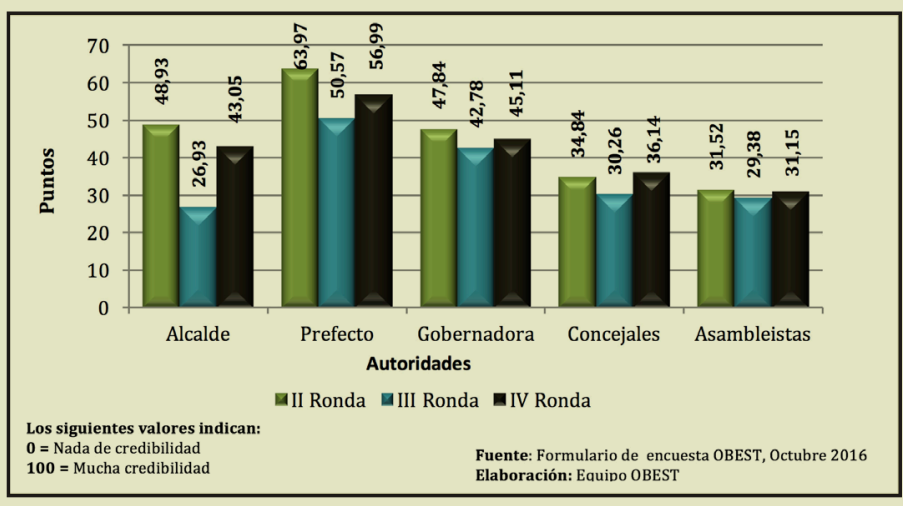

\section{Aspectos locales}

Se da esta definición a los servicios recibidos por los ciudadanos tales como: transporte público, suministro de servicios públicos, y control de velocidad por fotorradares. Además se presentan los actos delincuenciales más comunes del cantón Ambato.

\section{Calidad de suministros públicos}

El mayor peso porcentual en la escala "muy bueno" lo poseen los servicios de agua potable y alumbrado público con una frecuencia porcentual de $31 \%$ en ambos casos; en la escala "bueno" nuevamente se sitúa el suministro de agua potable con el 56\%, y en el caso del parqueo tarifado el 30\% de la población considera que es "regular" mientras que el 16\% piensa que es "malo".

\section{Tabla 5. Percepción ciudadana sobre el suministro de servicios básicos en la zona urbana del cantón Ambato (\% encuestados) Octubre 2016}

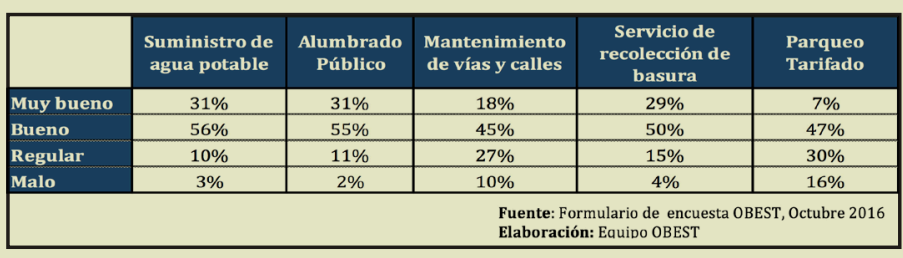

En cuanto al índice de percepción calculado, se muestran los siguientes resultados, el más alto de ellos es el suministro de agua potable con 71,55 puntos.

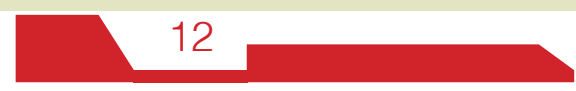


Fernando Mayorga, Tatiana Vayas, Carolina Freire, Lorena Rivera / Cuarto sondeo de opinión ciudadana de instituciones, autoridades y aspectos locales en la zona urbana del cantón Ambato

Figura 3. Índice de Percepción ciudadana sobre el suministro de servicios básicos en la zona urbana del cantón Ambato (escala: 0 a 100 puntos) II, III y IV ronda del sondeo

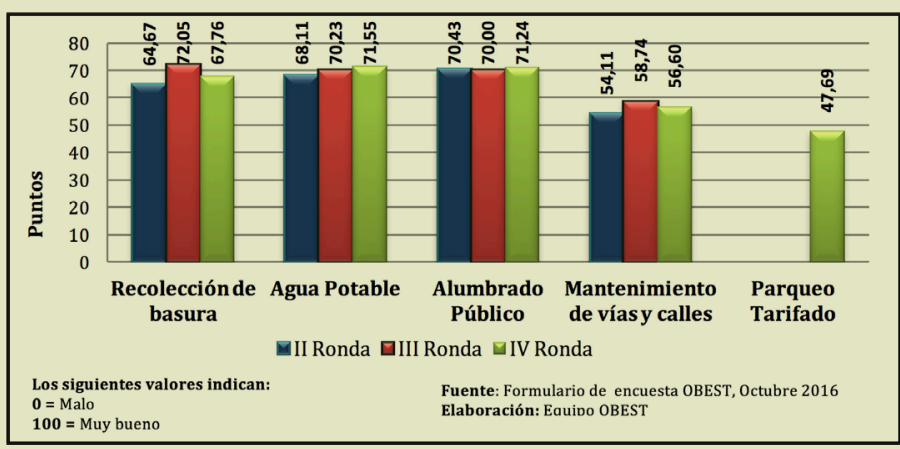

Al analizar los índices de percepción sobre el suministro de servicios básicos entre la tercera y cuarta ronda del sondeo, se evidencia que el suministro de agua potable y alumbrado público incrementan su índice mientras que la recolección de basura y el mantenimiento de vías y calles decrecen; es así que, el servicio de recolección de basura pasa del primer al tercer lugar (disminución de 4,29 puntos). En el segundo y tercer sondeo el servicio con menor puntuación fue el mantenimiento de vías y calles (54,11 y 58,74 puntos respectivamente), pero en el cuarto sondeo el parqueo tarifado es el menor puntuado (47,69 puntos).

\section{Calidad del transporte público}

Se consulta a los ciudadanos que residen en la zona urbana del cantón Ambato, sobre la calidad del servicio del transporte público tanto de buses como de taxis de manera independiente, para que seleccionen su percepción en función de las siguientes escalas: "muy bueno", "bueno", "regular" y "malo".

Tabla 6. Percepción ciudadana sobre la calidad del servicio de transporte público en la zona urbana del cantón Ambato (\% encuestados) Octubre 2016

\begin{tabular}{|l|c|c|}
\hline & Buses & Taxis \\
\hline Muy bueno & $\mathbf{8 \%}$ & $\mathbf{1 0 \%}$ \\
\hline Bueno & $\mathbf{5 1 \%}$ & $\mathbf{5 7 \%}$ \\
\hline Regular & $\mathbf{3 2 \%}$ & $\mathbf{2 6 \%}$ \\
\hline Malo & $\mathbf{9 \%}$ & $\mathbf{7 \%}$ \\
\hline \multicolumn{2}{|c|}{$\begin{array}{c}\text { Fuente: Formulario de encuesta OBEST, Octubre 2016 } \\
\text { Elaboración: Equipo OBEST }\end{array}$} \\
\hline
\end{tabular}

En general se observa que más de la mitad de los encuestados tienen una "buena" percepción del servicio que provee buses y taxis, sin embargo tres de cada diez personas consideran que el servicio de buses es "regular" y en el caso de taxis dos de cada diez. En cuanto a la percepción negativa, menos de la décima parte de los sujetos encuestados consideran que el servicio que brindan buses y taxis es "malo" (9\% y $7 \%$ respectivamente).

Al comparar el índice de percepción ciudadana de la calidad del servicio de buses y taxis entre la tercera y cuarta ronda del sondeo, se observa un crecimiento en ambos, siendo el servicio de taxis el que presenta un incremento mayor ( 9,71 puntos).

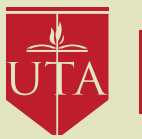

UNIVERSIDAD

TÉCNICA DE AMBATO
Figura 4. Índice de Percepción ciudadana sobre la calidad del servicio de transporte público en la zona urbana del cantón Ambato (escala: 0 a 100 puntos) III y IV ronda del sondeo

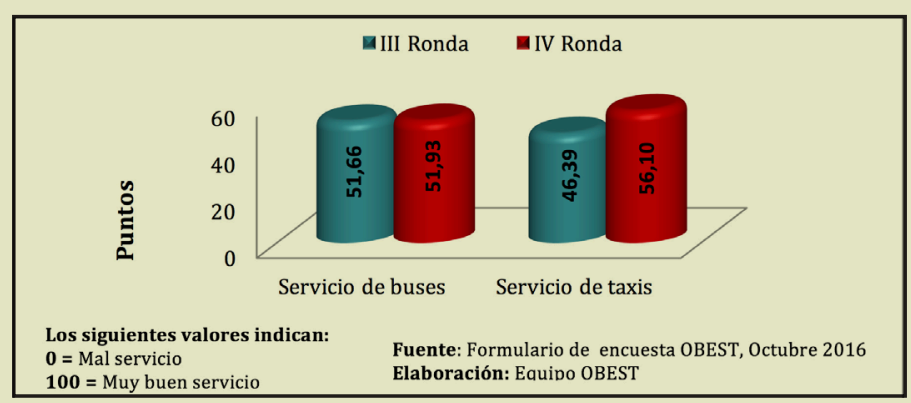

Percepción ciudadana del uso de fotorradares y seguridad vial

Se consulta a los pobladores locales su percepción respecto a la seguridad vial a partir de la implementación de los fotorradares en Ambato según las siguientes variables: accidentes de tránsito y personas que conducen en exceso de velocidad, con opciones de respuesta: "aumentaron", "se mantienen" y "disminuyeron".

Figura 5. Percepción ciudadana sobre el uso de fotorradares en cuanto a seguridad vial en la zona urbana del cantón Ambato (\% encuestados) Octubre 2016

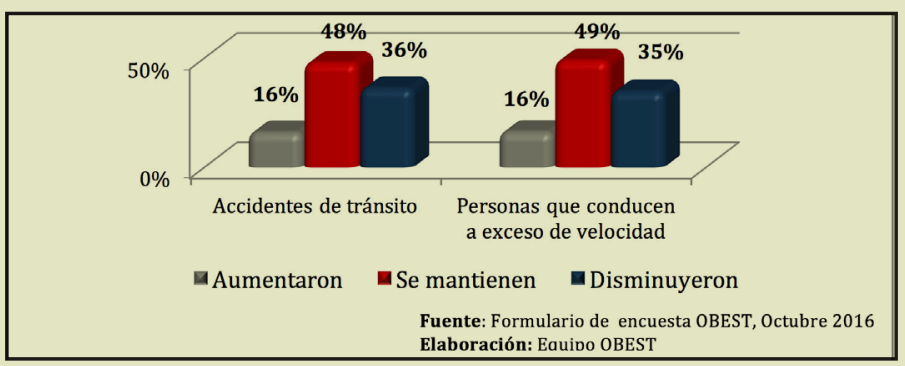

En el tercer y cuarto sondeo de opinión se mantiene el resultado en que cuatro de cada diez encuestados consideran que los accidentes de tránsito y las personas que conducen a exceso de velocidad se han mantenido a partir de la implementación de fotorradares en el cantón Ambato, sin embargo, la percepción positiva sobre la disminución de accidentes de tránsito pasa del 39\% al 36\%.

Adicionalmente se busca captar la opinión de los ciudadanos respecto a la influencia que tiene el uso de los fotorradares en el turismo local, obteniendo los siguientes resultados:

Figura 6. Percepción ciudadana sobre el uso de fotorradares en cuanto a turismo en la zona urbana del cantón Ambato (\% encuestados) Octubre 2016

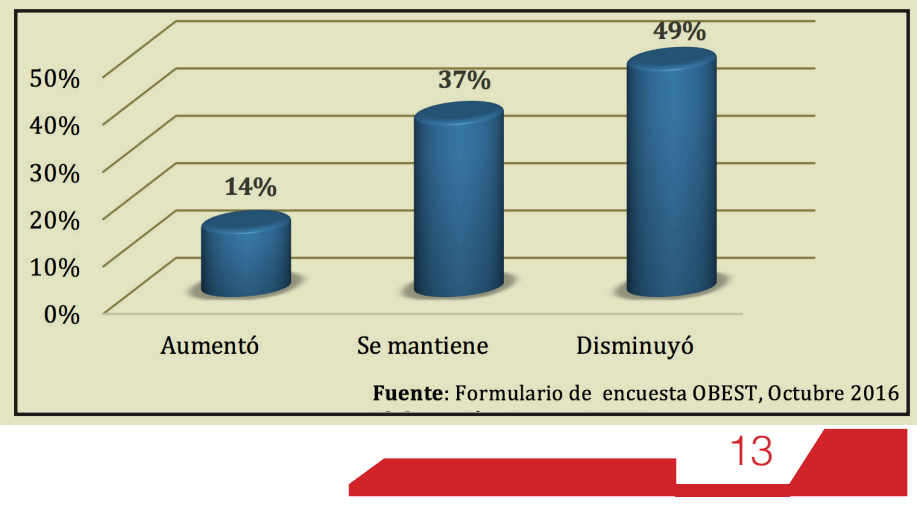


En el presente sondeo el $49 \%$ de los encuestados coincide de acuerdo a su percepción que a partir de la implementación de fotorradares en Ambato, el turismo ha disminuido en el cantón, frente al $69 \%$ del sondeo anterior, mientras que, las personas que creen que se ha mantenido pasa del $23 \%$ al $37 \%$; y los que consideran que el turismo ha aumentado sube del $8 \%$ al $14 \%$.

\section{Actos delincuenciales}

El objetivo principal de esta pregunta es identificar el tipo de acto delincuencial más común que sufren los ciudadanos del cantón Ambato de manera directa o indirecta como consecuencia de los delitos: amenaza, asalto/robo a personas, estafa, robo a vehículos o sus partes, robo a vivienda.

Figura 7. Víctimas de actos delincuenciales en los últimos 12 meses en la zona urbana del cantón Ambato (\% encuestados) Octubre 2016

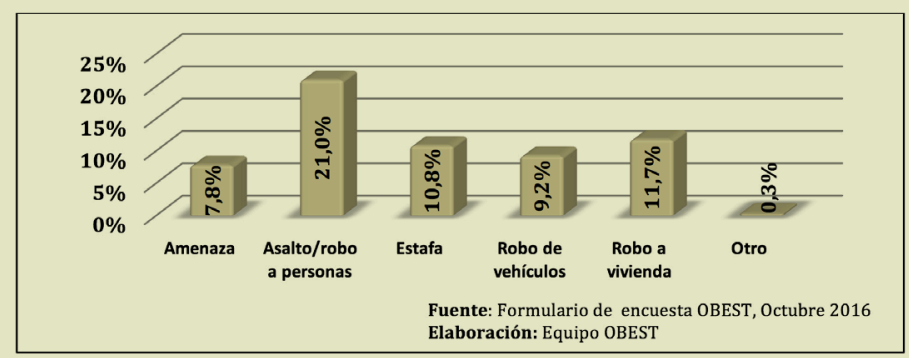

En la tercera y cuarta ronda del sondeo de opinión, se evidencia que 2 de cada 10 encuestados afirman haber sido víctimas de asalto/robo a personas en los últimos 12 meses, siendo éste el delito más frecuente. El robo de vehículos pasa del segundo al cuarto lugar como delito frecuente mientras que el robo a viviendas se ubica en segunda posición con 11,7\% de víctimas.

\section{Percepción de la imagen de las Universidades en el cantón Ambato}

Por la importancia que refiere la actividad académica en el desarrollo del país, se considera relevante conocer la opinión ciudadana sobre la imagen de las instituciones de educación superior locales: Universidad Regional Autónoma de los Andes - UNIANDES, Universidad

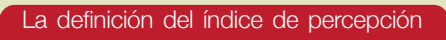
ciudadana sobre la imagen de las universidades locales, es igual al índice de percepción ciudadana sobre imagen institucional, es decir que no se evalúa su gestión o calidad, sino cómo las personas observan a la institución académica, indistintamente si han sido usuarios de las mismas. Indoamérica, Pontificia Universidad Católica del Ecuador Sede Ambato - PUCESA y Universidad Técnica de Ambato - UTA.

En la estructura de la pregunta se plantea cuatro opciones: "muy buena", "buena", "regular" y mala".

Tabla 7. Percepción ciudadana sobre la imagen de las universidades locales en la zona urbana del cantón Ambato (\% encuestados) Octubre 2016

\begin{tabular}{|l|c|c|c|c|}
\hline & UNIANDES & INDOAMÉRICA & PUCESA & UTA \\
\hline Muy buena & $13 \%$ & $10 \%$ & $28 \%$ & $46 \%$ \\
\hline Buena & $64 \%$ & $59 \%$ & $60 \%$ & $46 \%$ \\
\hline Regular & $21 \%$ & $27 \%$ & $10 \%$ & $7 \%$ \\
\hline Mala & $2 \%$ & $3 \%$ & $1 \%$ & $1 \%$ \\
\hline \multicolumn{4}{|c|}{$\begin{array}{c}\text { Fuente: Formulario de encuesta OBEST, Octubre 2016 } \\
\text { Elaboración: Equivo OBEST }\end{array}$} \\
\hline
\end{tabular}

De las universidades descritas, UNIANDES, PUCESA Y UTA son consideradas por los encuestados en su mayoría como "buenas" a excepción de la UTA que tiene una frecuencia porcentual de $46 \%$ en las opciones "muy buena" y "buena".

Figura 8. Índice de Percepción ciudadana sobre la imagen de las universidades locales en la zona urbana del cantón Ambato (escala: 0 a 100 puntos) II, III y IV ronda del sondeo

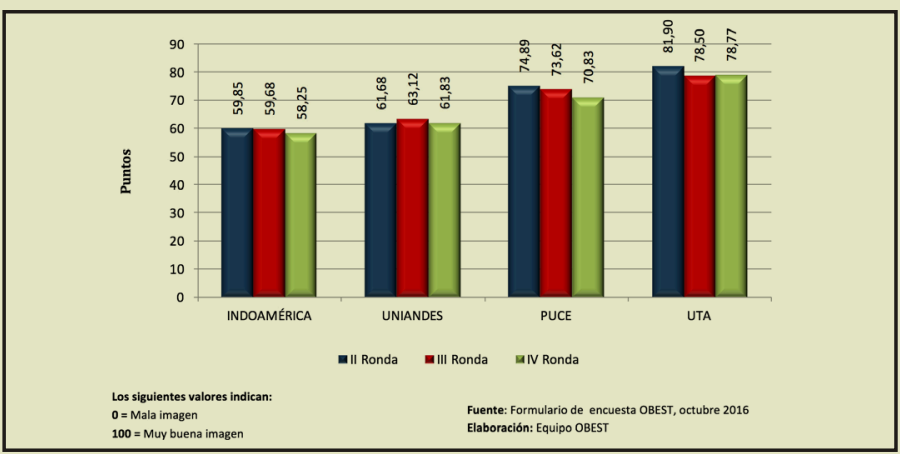

Los resultados registran las siguientes posiciones: primer lugar para UTA (78,77 puntos), segundo PUCESA (70,83 puntos), en tercer puesto UNIANDES (61,83 puntos) y en cuarta posición la Universidad Indoamérica (58,25 puntos).

Estos datos comparados con el tercer sondeo de opinión presentan variaciones decrecientes a excepción de la UTA que registra un leve incremento de 0,27 puntos; sin embargo, el ranking no se modifica.

\section{Conclusiones}

En este artículo, se evidencian los resultados obtenidos del cuarto sondeo de opinión ciudadana de instituciones, autoridades y aspectos locales del cantón Ambato; en el cual se aplicaron métodos por muestreo aleatorio simple, estratificado por zonas basado en el índice de confianza en investigaciones de México, El Salvador y Chile.

Los resultados muestran que la institución que proyecta una mejor imagen en los habitantes es la Empresa Eléctrica Ambato (EEASA) (70,46 puntos) seguido por el ECU 911 (68,09 puntos).

La autoridad con mayor credibilidad para los ciudadanos es el Prefecto de Tungurahua (56,99 puntos), seguido por la Gobernadora (45,11 puntos). Además, el Alcalde de Ambato ha tenido una recuperación significativa con respecto a los datos de la tercera ronda del sondeo (crecimiento de 16,12 puntos).

El servicio público con mejor puntuación, de acuerdo a la perspectiva de los encuestados, es el agua potable (71,55 puntos), seguido por el alumbrado público (71,24 puntos). Además, la calidad del servicio de transporte público refleja una recuperación notoria, en relación al sondeo anterior, especialmente en taxis.

La mayoría de los habitantes de la zona urbana consideran que, desde la implementación de los fotorradares, el turismo ha disminuido en la ciudad (49\% de encuestados); mientras que, los accidentes de tránsito y las personas que conducen a exceso de velocidad se han mantenido (48\% y $49 \%$ de encuestados, respectivamente).

El acto delincuencial con mayor frecuencia en Ambato en los últi- 
mos 12 meses, según sus habitantes, es el asalto/robo a personas (21\% de encuestados), seguido por robo a viviendas $(11,7 \%$ de encuestados).

La institución de educación superior que muestra una mejor imagen de acuerdo a la opinión de la población es la UTA (78,77 puntos), manteniéndose en primer lugar desde la tercera ronda del sondeo.
Los resultados de este trabajo contribuyen como herramienta para la toma de decisiones por parte de los gestores de desarrollo de la provincia de Tungurahua; además, mantiene informada a la ciudadanía ambateña de aspectos locales de su cantón.

\section{Anexo}

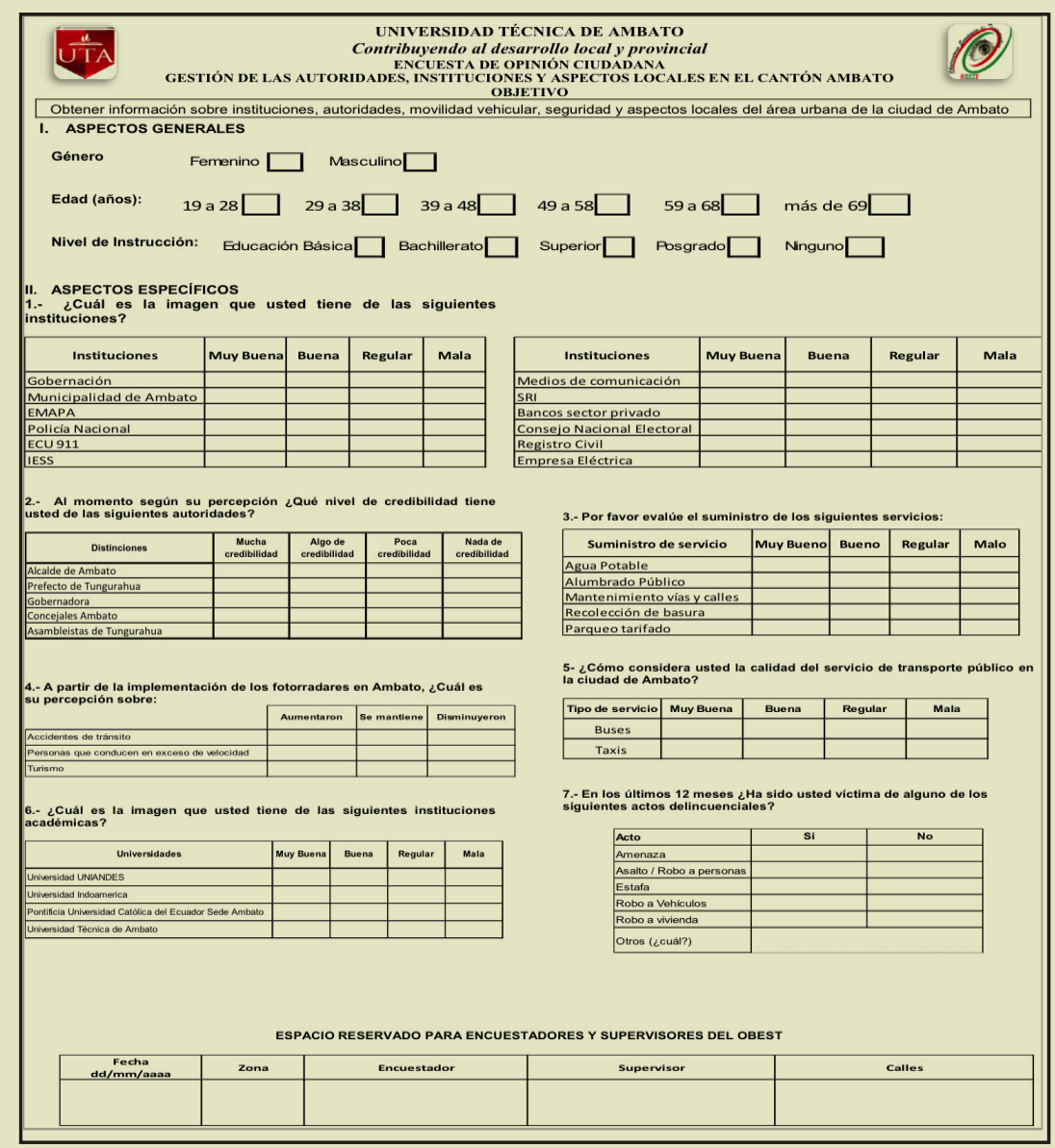

\section{Referencias}

- Aguilar, J. (2013). La percepción de la seguridad y la confianza en instituciones públicas. Línea de base del plan de acción asocio para el crecimiento. San Salvador, El Salvador: Instituto Universitario de Opinión Pública, de la Universidad Centroamericana José Simeón Cañas.

- Burgoa, L. (1995). Palabras y creencias. Ensayo crítico acerca de la comunicación humana y de las creencias. Murcia, España: Secretariado de Publicaciones, Universidad de Murcia.

- IBOPE inteligencia. (2011). Índice de confianza social Chile - 1era medición.

- Moreno, A. (2010). Confianza en las instituciones. México en perspectiva comparada. México, D. F., México: Centro de Estudios Sociales y de Opinión Pública. Cámara de Diputados / LXI Legislatura.

- Muñoz, M. (2010). Protocolo y relaciones públicas. Madrid, España: Ediciones Paraninfo, S.A.

- Sánchez, R. (2014). Gestión y psicología en empresas y organizaciones. Madrid, España: ESIC editorial.

\section{Coordinación General}

Econ. Diego Proaño C., Mg.

\section{Análisis, diseño e interpretación} Equipo Técnico OBEST

\section{Planificación de trabajo de campo}

Equipo Técnico OBEST

\section{Levantamiento de información}

Supervisión equipo técnico OBEST

\section{Encuestadores OBEST}

Equipo de encuestadores de la Facultad de Contabilidad y Auditoría de la UTA

Equipo de encuestadores de la Facultad de Jurisprudencia y Ciencias Sociales de la UTA

\section{Colaboración}

Ing. Jorge Tamayo Viera

Econ. Nelson Lascano 